\section{Voz e fala no menacme e na pós-menopausa}

\section{Voice and speech during menacme and postmenopause}

\author{
Eliséa Maria Meurer 1 \\ Maria Celeste Osório Wender 2 \\ Helena von Eye Corleta 3 \\ Edison Capp 4
}
1 Departamento de Fisiologia. Universidade Federal do rio Grande do Sul, Porto Alegre, RS, Brasil.
2-4 Departamento de Ginecologia e Obstetrícia. Hospital de
Clínicas de Porto Alegre, Faculdade de Medicina.
Universidade Federal do Rio Grande do Sul. Rua Ramiro Barcelos, 2400, $4^{\circ}$ andar, Porto Alegre, RS, Brasil. CEP: 90.035-003

\begin{abstract}
Objectives: to compare phono-articulatory characteristics between women in reproductive age and postmenopause women.

Methods: acoustic variations in vocal intonation, speed of the speech and the pause pattern were measured. Fourty five reproductive age women with regular menstrual cycles and taking no hormonal contraceptives and 45 postmenopause women receiving no hormonal replacement therapy for at least three years were interviewed and their verbal productions were recorded. Acoustic analyses were performed using the Kay Elemetrics Motor Speech Profile. Student's t test was employed to compare data between the two groups when they presented normal distribution, and Mann-Whitney test when they were asymmetrical.

Results: the reduction on the rhythm and phonoarticulatory speed was more constant in the postmenopause group.

Conclusions: a better understanding in this field will make possible to elaborate strategies to offer a better life quality for postmenopausal women, in special for those who use their voice professionally.
\end{abstract}

Key words Voice, Postmenopause, Speech acoustics

\section{Resumo}

Objetivos: comparar as características fonoarticulatórias de mulheres no menacme e na pósmenopausa.

Métodos: foram verificados parâmetros acústicos em tons de voz, sua estabilidade, modulações de entonações, ritmo e velocidade de fala. Quarenta e cinco mulheres na pós-menopausa há três anos ou mais e sem terapia de reposição hormonal e, quarenta e cinco mulheres no menacme, com ciclos menstruais regulares e sem medicação hormonal responderam um questionário e gravaram produções verbais. As análises acústicas foram realizadas com o programa Motor Speech Profile da Key Elemetrics. Utilizou-se o teste t de Student para resultados com distribuição gaussiana e o teste Mann-Whitney quando houve distribuição assimétrica.

Resultados: no grupo pós-menopausa houve maior consistência na lentificação do ritmo e redução de velocidade fonoarticulatória.

Conclusões: o melhor conhecimento nesta área permitirá a elaboração de estratégias para oferecer melhor qualidade de vida para mulheres na pósmenopausa, em especial para aquelas que utilizam a voz profissionalmente.

Palavras-chave Voz, Pós-Menopausa, Acústica da fala 


\section{Introdução}

A expressividade humana é modificada de acordo com a intenção do que se quer transmitir. Suspiros, riso, bocejos, tosse, combinações de vogais e consoantes emitidas nas palavras e frases dos diversos idiomas e, o canto, podem ser recursos de comunicação. Todas essas manifestações têm como traço sonoro comum variações de modulações, ritmo e velocidade. Suas propriedades acústicas, decompostas em freqüência, medida em hertz $(\mathrm{Hz})$, intensidade, medida em decibel (dB) e, duração, medida em segmentos por segundo (s) e milissegundos (ms) possibilitam conhecer processos neurofisiológicos da produção motora de fala, ou fonoarticulação. 1

Em relações interpessoais e profissionais, a competência comunicativa de mulheres também recebe influências dos efeitos secundários cíclicos de esteróides. Dessa forma, já foram relatados períodos típicos e outros, mais individualizados de agravamento vocais, temporários ou permanentes, no ciclo vital feminino.

O período dos 25 aos 40 anos de vida é considerado de melhor performance vocal, 2 abrange a etapa dos 30 aos 40 anos, em que há tendência de redução na duração dos ciclos menstruais. ${ }^{3}$ Nesta fase, ocorrem as primeiras modificações foliculares que evoluirão para a cessação da capacidade reprodutiva feminina. Para mulheres na pós-menopausa, os sintomas de agravamento da voz, redução de microestabilidade vocal, das variações de tons, ${ }^{4}$ ocorrem concomitantes com os calorões, problemas cardiovasculares, incontinência urinária, osteoporose, depressão e demência. 5 Cantoras líricas demonstraram maior percepção das modificações vocais. ${ }^{6}$ Terapias de reposição hormonal podem contribuir para acentuar a virilização vocal, ou, para favorecer o retorno a padrões anteriores à menopausa. ${ }^{4}$ Os efeitos de esteróides na pós-menopausa, que incluíram emissões encadeadas de vogais e consoantes na fala, articulação verbal e fluência, estão mais relacionados à cognição e demência.7-11

O objetivo desta pesquisa foi verificar características acústicas fonoarticulatórias no tom de base vocal, sua estabilidade, modulações da voz, agilidade articulatória, ritmo e velocidade de fala, de mulheres no menacme e na pós-menopausa.

\section{Métodos}

O cálculo do tamanho da amostra foi realizado a partir de estudo piloto com 30 mulheres, para um poder estatístico de $80 \%$ e intervalo de confiança de 95
(IC95\%). Entre julho e setembro de 2001, foram pesquisadas 90 mulheres (45 no grupo menacme e 45 no grupo pós-menopausa) atendidas no Ambulatório de Ginecologia do Hospital de Clínicas de Porto Alegre (HCPA), da Universidade Federal do Rio Grande do Sul, Brasil, e, voluntárias da comunidade local. Nenhuma mulher referiu tratamentos hormonais nem outras limitações orgânicas, neurológicas, cognitivas, ou emocionais. Foram incluídas mulheres não fumantes e sem treino vocal para o canto ou a fala. Os critérios para inclusão no grupo menacme (30 a 40 anos de idade), foram regularidade de ciclos menstruais e ausência de consumo de contraceptivos orais. Para inclusão no grupo pósmenopausa, foi requerida menopausa há três anos ou mais e ausência de terapias de reposição hormonal (TRH). Este projeto foi aprovado pelo Comitê de Ética do HCPA, da Universidade Federal do Rio Grande do Sul.

\section{Procedimentos}

Após esclarecimento detalhado sobre objetivos, vantagens e riscos do projeto, as voluntárias assinaram "Termo de Consentimento". Essas preencheram questionário com informações sobre suas vozes, fala, boca, dieta, outros hábitos, situação ginecológica e fatores associados.

Registros acústicos foram coletados com microfone Shure, modelo $16 \mathrm{~A}$, gravador digital Sony MZ-R7-S1 e, minidiscos Sony 74. O microfone polar, cardióide, unidirecional, possuía banda plana entre 50 e $15000 \mathrm{~Hz}$ com pico de variação de captura inferior a $4 \mathrm{~dB}$ entre 6000 e $7000 \mathrm{~Hz}$, para preservar maior fidedignidade nas gravações. Esse microfone foi colocado a uma distância de $10 \mathrm{~cm}$, em frente à boca das pesquisadas, que permaneceram em pé durante as gravações, para favorecer seus processos fonoarticulatórios. Os registros foram repetidos sempre que elas desejaram treinar e, até constatar-se que os sensores do gravador marcavam padrões analisáveis. Nas análises utilizou-se o programa Motor Speech Profile (MSP), acoplado ao sistema Kay Elemetrics Computerized Speech Laboratory (CSL modelo 4341).

Foram gravados 15 segundos de ruído ambiental, para controle de interferências deste nos registros das pesquisadas. A seguir, coletaram-se emissões da vogal/a/prolongada por 1,5 segundos, para verificar o tom fundamental, ou de base da voz $\left(\mathrm{F}_{\mathrm{o}}\right)$, mais agudo (Frhi), mais grave (Frlo) e sustentação vocal (std). Entre cinco repetições da diadococinesia ver$\mathrm{bal} / \mathrm{pa}$ ta $\mathrm{ka} / \mathrm{gravados}$, foram analisados os $2^{\circ}, 3^{\circ} \mathrm{e} 4^{\circ}$ segmentos, para obter-se ritmo (padrão DDK), 
velocidade (veloc DDK) e oscilações (desvio DDK) articulatórios, da mesma forma que para constatar-se intensidade, ou volume da voz (padrão intensidade) e suas variações (variação de intensidade). Com a emissão em tom neutro da frase "irei a Gramado nas férias de inverno", registrou-se a freqüência fundamental de fala (FFF), a mais alta (FFrhi), a mais baixa (FFrlo) e as variações de entonações (Fstd). A mesma frase foi analisada na velocidade de fala (velocidade silábica), duração de sílabas (duração silábica) e, duração de pausas (padrão pausal).

\section{Análise estatística}

Os dados com distribuição normal foram comparados pelo teste $t$ de Student (Fo, Frhi, Frlo, FFF, FFrlo). O teste "U" de Mann Whitney foi utilizado nos resultados em que não houve distribuição gaussiana (std, padrão DDK, veloc DDK, desvio DDK, padrão de intensidade, variação de intensidade, FFrhi, FFrlo, Fstd, velocidade silábica, duração silábica, padrão pausal).

\section{Resultados}

A idade média das mulheres do grupo menacme foi $35,61 \pm 3,25$ anos e os ciclos menstruais tiveram regularidade de $28 \pm 3$ dias. No grupo de mulheres na pós-menopausa, a idade média foi de $62 \pm 7,42$ anos e, tendo ausência de mestruação de 13,95 \pm 8,32 anos.
Ficou constatada a inexistência de acréscimo de ruído ambiente nos registros gravados. Os resultados apresentados na Tabela 1 mostram ausência de agravamento vocal na emissão prolongada do monotom /a/, no grupo pós-menopausa, por terem inexistido diferenças estatísticas significativas nos tons fundamentais (Fo), mais agudos (Fhi) e mais graves (Flo), das mulheres dos dois grupos. Os resultados de estabilidade vocal (std), da mesma Tabela 1, mostraram que houve diferença estatística significante $(\mathrm{p}=0,045)$.

Na Tabela 2, observa-se que as mulheres pósmenopausa utilizaram tempo mais longo (padrão DDK), na produção da diadococinesia verbal $(\mathrm{p}=0,001)$ e apresentaram menor velocidade de fala (velocidade DDK), do que as do grupo menacme $(p=0,001)$. Na mesma tabela verifica-se que também não houve diferença estatística os padrões de volume (padrão de intensidade) e, nas flutuações de energia sonora (variação de intensidade) entre os grupos.

$\mathrm{Na}$ Tabela 3, para análises da emissão da frase expressa com entonação neutra, observa-se que não houve diferenças estatísticas significativas entre os grupos de mulheres, no tom de fala (FFF), mais alto (FFrhi), mais baixo (FFrlo) e nas variações de tons (Fstd). Mulheres na pós-menopausa apresentaram menor velocidade de fala $(p=0,005)$. No ritmo de fala, as pausas, intercaladas com as produções sonoras, também foram maiores no grupo pós-menopausa $(\mathrm{p}=0,028)$.

Tabela 1

Registros acústicos de monotom na vogal /a/sustentada.

\begin{tabular}{lccc}
\hline & Menacme & Pós-menopausa & $p$ \\
\hline$F_{\circ}(\mathrm{Hz})^{*}$ & $206,58 \pm 19,43$ & $201,92 \pm 29,40$ & 0,377 \\
Frhi $(\mathrm{Hz})^{*}$ & $213,98 \pm 20,75$ & $210,74 \pm 31,71$ & 0,569 \\
Frlo $(\mathrm{Hz})^{*}$ & $198,29 \pm 21,24$ & $188,79 \pm 33,98$ & 0,116 \\
Std $(\mathrm{Hz})^{* *}$ & $2,73(0,79-3,01)$ & $3,56(1,50-49,13)$ & 0,045 \\
\hline
\end{tabular}

$F_{\mathrm{o}}=$ fom fudanmental basal; $\mathrm{Hz}=$ Hertz; Frhi = tom mais agudo; Frlo = tom mais grave; Std = sustentação vocal; * média \pm desvio padrão, ** mediana (intervalo interquartis) 
Tabela 2

Registros acústicos da diadococinesia /PA TA KA/

\begin{tabular}{lrrr}
\hline & \multicolumn{1}{c}{ Menacme } & Pós-menopausa & \multicolumn{1}{c}{$\boldsymbol{p}$} \\
\hline Padrão DDK (ms)* & $158,70(127,83-371,89)$ & $227,63(128,25-580,20)$ & 0,001 \\
Velocidade DDK $(/ \mathrm{s})^{*}$ & $6,30(2,69-7,82)$ & $4,42(1,72-7,80)$ & 0,001 \\
Desvio DDK (ms)* & $52,14(9,56-289,02)$ & $51,86(14,44-296,60)$ & 0,360 \\
Padrão de intensidade $(\mathrm{dB})^{*}$ & $63,47(56,74-73,10)$ & $64,00(56,56-78,30)$ & 0,225 \\
Variação de intensidade $(\mathrm{dB})^{*}$ & $1,46(0,61-9,48)$ & $1,77(0,48-9,56)$ & 0,750 \\
\hline
\end{tabular}

$\mathrm{F}_{\mathrm{o}}=$ fom fudanmental basal; $\mathrm{Hz}=$ Hertz; Frhi = tom mais agudo; Frlo = tom mais grave; Std = sustentação vocal; * média \pm desvio padrão, ** mediana (intervalo interquartis)

Tabela 3

Registros acústicos da frase "irei a Gramado nas férias de inverno" emitida com entonação neutra.

\begin{tabular}{lrrr}
\hline & \multicolumn{1}{c}{ Menacme } & Pós-menopausa & $\boldsymbol{p}$ \\
\hline FFF (Hz)* & $195,53 \pm 23,74$ & $188,38 \pm 18,17$ & 0,112 \\
FFrlo (Hz)* & $146,24 \pm 31,30$ & $134,40 \pm 27,22$ & 0,059 \\
FFrhi (Hz)** & $238,70(200,30-506,58)$ & $243,72(193,64-508,68)$ & 0,634 \\
Fstd (Hz)** & $21,06(8,71-70,10)$ & $23,15(11,44-56,56)$ & 0,204 \\
Velocidade silábica (/s)** & $4,74(2,48-10,28)$ & $3,79(2,24-9,77)$ & 0,005 \\
Duração silábica (ms) ** & $188,94(89,30-291,10)$ & $203,13(102,40-321,89)$ & 0,150 \\
Padrão pausal (ms) ** & $146,14(0,00-705,63)$ & $166,62(0,00-925,07)$ & 0,028 \\
\hline
\end{tabular}

FFF = tom da fala; $\mathrm{Hz}=$ Hertz; Ffrhi = tom mais agudo; Ffrlo = tom mais grave; Fstd = variações nos tons silábicos; $\mathrm{ms}=$ milisegundos; * média \pm desvio padrão, ** mediana (intervalo interquartis)

\section{Discussão}

A ausência de diferenças estatísticas significantes na emissão da voz no monotom foi diferente das citações que mostram a virilização vocal como um dos sintomas fonatórios mais comuns após a cessação dos ciclos menstruais. $6 \mathrm{O}$ agravamento da voz, para Abitbol et al., 4 é decorrente dos efeitos secundários locais de esteróides, sobre cartilagens, músculos, mucosas e lubrificação dos tecidos laríngeos. Os efeitos hormonais na massa e rigidez, associados ao alongamento do trato vocal resultariam na redução de freqüência fundamental da voz. ${ }^{1}$

$\mathrm{Na}$ idade média das mulheres do grupo pósmenopausa, já teria ocorrido a finalização do agravamento vocal, característico do ciclo do envelhecimento feminino. 12 O período de estacionamento do tom vocal agravado pela senilidade vocal, ou presbi- fonia e, pela menopausa, que fora constatado em estudos feitos com pessoas de idades até em torno de 90 anos, não foi relacionado ao uso ou não de medicações hormonais das mulheres. 2,13

A variação de microestabilidade vocal, mais acentuada nas mulheres pós-menopausa, não evidenciou superação dos limiares sonoros de 4 a $8 \mathrm{~Hz}$, que ainda caracterizariam um parâmetro de flutuação natural, na voz humana. ${ }^{1}$ Da mesma forma, as oscilações na sustentação vocal, com duração inferior a um terço do tempo de emissões, não equivaleram a tremor vocal. A alteração da manutenção da eficiência de regulação dos movimentos rítmicos, involuntários, decorrentes de descontração de grupos musculares, desencadearia o tremor vocal. 14 A coordenação motora fina verbal realizada por estruturas como o cerebelo, já foi descrita como influenciada por estrógenos, mas também, por andrógenos. 15 
Esses resultados também diferiram de redução da microestabilidade vocal, a qual consistiria no outro sintoma mais relacionado com a pós-menopausa. 4 Refletiram a maior variabilidade intraindividual, freqüente no envelhecimento humano. ${ }^{12,13}$

A existência de diferenças estatísticas no ritmo e na velocidade da diadococinesia verbal demonstrou decréscimo na mobilidade articulatória, do grupo pós-menopausa. Esse processo requer interações dinâmicas corticais, subcorticais e de diversos nervos cranianos.16,17 Nas interações corticais e subcorticais, as modificações poderiam ter decorrido de variações estruturais e metabólicas do envelhecimento, 18 fadiga e debilidade por disfunções musculares difusas, ou da supressão de estrógenos.19,20 A intensidade vocal foi semelhante nos dois grupos e em outros estudos feitos com mulheres jovens. 21,22

A ausência de diferença estatística significante entre os grupos, nos itens da flutuação do ritmo de fala e da intensidade vocal, possibilita inferir que a lentificação da mobilidade articulatória não acarretou descontrole da habilidade de mover articuladores supraglóticos, na produção de seqüências sonoras. O mesmo ocorreu na regularidade de emissão das sílabas, para o grupo pós-menopausa. Ficou expressa a conservação da habilidade do grupo pósmenopausa em coordenar energia, pressão e amplitude do fluxo expiratório e ciclos vibratórios laríngeos. 23

A ausência de diferenças estatísticas entre os grupos de mulheres nos tons de fala da frase neutra, demonstrou que elementos inseridos no contexto comunicativo como os graus de escolaridade e frequiência de contato com público, foram padronizados nos processos fonoarticulatórios. Atribuiu-se essa ocorrência ao treino prévio às gravações, que teve como efeito assegurar a chance de todas as mulheres demonstrarem melhores competências motoras comunicativas.

Ambos os grupos de mulheres também se assemelharam ao comportamento de aproximarem o tom fundamental de fala da frase ao tom mais grave da emissão da voz sustentada (Frlo) e não, ao tom de base (Fo) deste monotom. Reproduziram a tendência de utilização de tons mais baixos, com menos variações, em frases de menor expressividade afetiva e lingüística, 14 o que ocorrera na sentença emitida com entonação neutra.
A redução de velocidade de fala na emissão da frase do grupo pós-menopausa, coincidiu com resultados da velocidade da diadococinesia verbal. Demonstrou lentificação nos ajustes da energia sonora e de articuladores ativos do trato supraglótico, durante as emissões mais básicas e nas mais complexas. Esses sugeriram modificação nos processos motores ou neurofisiológicos. A regularidade nas emissões das unidades sonoras da frase foi acompanhada de prolongamentos nos intervalos, ou silêncios comunicativos, entre palavras. Essas pausas no enunciado verbal não informariam sobre possíveis efeitos da idade sobre a fonoarticulação, 13 mas, atribuídas a fatores neuromotores sob controle do Sistema Nervoso Central,24 permitiram estimar que decorreram de desajustes motores. O prolongamento das pausas no grupo pós-menopausa, inferior a um segundo cada, não caracterizou perda de fluência motora, definida pela American Speech-Hearing Association (ASHA) como aspecto da produção da fala referente à continuidade, suavidade e velocidade, com ou sem esforço. 25

\section{Conclusões}

Na pós-menopausa ocorreu lentificação do ritmo e redução de velocidade de fala, além de perda da microestabilidade vocal. Embora essas alterações relacionadas à regulação neurofisiológica da fonoarticulação possam ser decorrentes das alterações hormonais que ocorrem entre o menacme e a pósmenopausa, neste estudo não foi possível afastar a possibilidade de serem também decorrentes do processo de envelhecimento humano. Esses resultados alertam para a necessidade de se valorizar as variações da fonoarticulação feminina no ciclo vital, com especificações de fases e de características de efeitos secundários hormonais, de tipos de competência de comunicação motora, cognitiva, ou afetiva, e, falada ou cantada. Para avaliação das modificações vocais e da fala antes e após a menopausa, o ideal seria o estudo de mesmos indivíduos nesses dois momentos do ciclo vital (avaliação longitudinal). O melhor conhecimento permitirá a elaboração de estratégias para oferecer melhor qualidade de vida para mulheres na pós-menopausa, que podem ter extensa longevidade nessa condição. 


\section{Agradecimentos}

Agradecemos o apoio financeiro do Fundo de Incentivo à Pesquisa do Hospital de Clínicas de Porto Alegre.

\section{Referências}

1. Russo ICP. Bases físicas da fonação. Acústica e psicoacústica aplicadas a fonoaudiologia. São Paulo: Lovise; 1999.

2. Behlau MS. Presbifonia: envelhecimento vocal inerente à idade. In: Russo IP. Intervenção fonoaudiológica na terceira idade. Rio de Janeiro: Revinter; 1999. p. 21-5.

20. Morrison JH, Hof PR. Life and death of neuron in the aging brain. Science 1997; 278: 412-9.

3. Spritzer PM, Mallmann ES. Ciclo menstrual. In: Oliveira HC, Lemgruber Z. Tratado de ginecologia: FEBRASGO Rio de Janeiro: Revinter; 2000. p. 231-6.

4. Abitbol J, Abitbol P, Abitbol B. Sex hormone and the female voice. J Voice 1999; 3: 424-46.

5. Greendale AR, Lee NP, Arrioila ER. The menopause. Lancet 1999; 353: 571-80.

6. Boulet MJ, Oddens BJ. Female voice changes around and after menopause-an inicial investigation. Maturitas 1996; 1: 15-21.

7. Shaywitz SE, Shaywitz BA, Pugg KR, Fulbrigth RK, Skudiersi P, Mencl WE, Constable RT, Naftolin F, Palker SF, Marchione KE, Katz L, Shcnkweiler DP, Fletcher JM, Lacadie C, Kelta M, Gore JC. Effect of estrogen on brain activation patterns in postmenopause women during working memory tasks. Obstet Gynecol Surv 2000; 55: $227-8$

8. Drake EB, Henderson VW, Stanczyk FZ, McCleary CA, Brown WS, Smith CA, Rizzo AA, Murdock GA, Buckwalter JG. Associations between circulating sex steroid hormones and cognition in normal elderly women. Neurology 2000; 54: 599-603.

9. Yaffe K, Sawaya G, Lieberburg I, Grady D. Estrogen therapy in postmenopausal women: effects on cognitive function and dementia. JAMA 1998; 279: 688-95.

10. Smith YR, Giordani B, Lajiness-O'Neill R, Zubieta JK. Long-term estrogen replacement is associated with improved measures in postmenopausal women. Fertil Steril 2001; 76:1101-7.

11. Le Blanc E, Janowsky J, Chan BKS, Nelson HD. Hormone replacement therapy and cognition-review and metaanalysis. JAMA 2001; 285: 1489-99.

12. Decoster W, Debruyne F. The aging of voice: changes in fundamental frequency, waveform stability and spectrum. Acta Otorhinolaryngol Belg, 1997; 51: 105-12.

13. Carbonell J, Tolosa F, Juan E. Prebyphonia: a study of acoustic parameters or normalcy. Acta Otorrinolaringol Esp 1996; 47: 295-9.

14. Botini A, Granström B, Möbius B. Developments and paradigms in intonation research. Speech Commun 2001; 33: 263-96.

15. Manolagas SC, Kousteni S. Nonreproductive sites of action of reproductive hormones. Endocrinology 2001; 142: 2200-4.

16. Crucian GP, Berenbaum SA. Sex differences in right hemisfere tasks. Brain Cogn 1998; 36: 377-89.

17. Gur RC, Alsop D, Glahn D, Petty R, Swanson CL, Maldjian JA, Turetsky BI, Detre JA, Gee J, Gur RE. An FMRI study of sex differences in regional activation to a verbal and a spatial task. Brain Lang 2000; 74: 157-70.

18. Murphy DGM, De Carli C, McIntosh AR, Daly E, Mentis MJ, Pietrini P, Szczepanik J, Shapiro MB, Grady CL, Horwitz B, Rapport S. Sex differences in human brain morphometry and metabolism: an in vivo. JAMA 1996; 53: $585-94$

19. Genazzani AR, Someti M, Rubino S, Bersi C, Spinetti A, Salvestroni C, Luisi M, Petraglia F. Neuroencrine changes and climaterium. Maturitas 1997; 27: 1-10.

20. Morrison JH, Holf PR. Life and death of neurons in the aging brain. American association for the advancement of science 1997, 278, (5337): 412-9.

21. Orlikoff RF. Vocal stability and vocal tract configuration: an acoustic and electroglottographic investigation. J Voice 1995; 9: 173-81.

22. Gelfer MP, Young SR. Comparisons of intensity measures and their stability in male and female speakers. J Voice 1997; 2: 178-86.

23. Lofqvist A, Lindblom B. Speech motor control. Curr Opin Neurobiol 1994; 4: 823-6.

24. Adams SG, Page AD. Effects of practice and feedback variabels on speech motor learning. J Med Speech-Leng Pathol 2000; 8: 215-20.

25. Sassi FC, Ostiz HC, Andrade CF. Terminologia: fluência e desordens da fluência. Pró-fono 2001; 13: 107-13.

Recebido em 8 de março de 2004

Versão final reapresentada em 30 de junho 2004

Aprovado em 4 de agosto de 2004 